\title{
A numerical approach to quasi-ballistic transport and plasma oscillations in junctionless nanowire transistors
}

\author{
Maziar Noei ${ }^{1} \cdot$ Tobias Linn $^{1} \cdot$ Christoph Jungemann ${ }^{1}$
}

Published online: 19 April 2020

(c) The Author(s) 2020

\begin{abstract}
A numerical framework for DC and RF small-signal simulations of nanowire transistors is presented, which is based on the self-consistent solution of the Poisson, Schrödinger, and Boltzmann transport equations and is stable for the entire range from weak to strong particle scattering. The proposed approach does not suffer from the deficiencies due to the transformation of the Boltzmann transport equation into the energy space and can handle the quasi-ballistic case. This is a key requirement for the investigation of plasma resonances and other high-mobility phenomena. The in-house solver is validated with results of a previously developed simulator based on the $H$-transformation for a conventional $\mathrm{N}^{+} \mathrm{NN}^{+}$silicon transistor with strong scattering. Then, its results are compared with those of moments-based models and it is shown that these do not provide a satisfactory description of the electron dynamics in the quasi-ballistic transport regime. Furthermore, the internal boundary conditions of the transport models at the contacts are found to have a significant impact on plasma resonances and the physics-based thermal-bath boundary condition strongly suppresses them.
\end{abstract}

Keywords Boltzmann equation $\cdot$ Nanowire transistor $\cdot$ Numerical simulation $\cdot$ Ballistic transport $\cdot$ Plasma oscillations

\section{Introduction}

Recently, scientific interest in solid-state terahertz (THz) devices (operating at frequencies between $300 \mathrm{GHz}$ and $3 \mathrm{THz}$ ) has increased significantly [1-5]. The main idea, based on the shallow-water analogy put forward by Dyakonov and Shur [6-8], is to use field-effect transistors with high-mobility channels as nonlinear elements for the resonant detection of incident $\mathrm{THz}$ radiation. Moreover, the possibility of plasma instabilities leading to the emission of $\mathrm{THz}$ radiation by planar field-effect transistors, as pointed out by Dyakonov and Shur, has resulted in investigations by many groups (e.g., [9-13]). The question arises whether similar plasma instabilities could occur in nanowire or nanotube transistors. Although the methods developed previously for planar transistors could be applied to nanowire transistors, it is not clear whether the assumptions on which these models are based hold in the case of quasi-ballistic transport, which is a prerequisite for plasma instabilities [14]. Therefore,

Maziar Noei

mn@ithe.rwth-aachen.de

1 Chair of Electromagnetic Theory, RWTH Aachen University, 52056 Aachen, Germany we discuss in addition to the drift-diffusion (DD) model, which is similar to the transport model used by Dyakonov and Shur, also the Boltzmann transport equation (BE) from which the DD model can be derived under rather stringent approximations [15].

Since the stabilization scheme based on the $H$-transformation for the BE fails for quasi-ballistic transport, a scheme based on the phase-space trajectories of the electrons is presented [16]. Furthermore, in the case of quasi-ballistic transport the stationary drain current of a MOSFET is limited by the finite injection velocity of the electrons from the source into the channel (e.g., $[17,18])$. This leads to a much more complex behavior of the source contact at high frequencies that can be described by the simple approximation made by Dyakonov and Shur and that the small-signal electron density must satisfy a Dirichlet boundary condition (D-BC) at the source side of the channel [6]. In order to describe the quasi-ballistic transport in such devices, it is not only necessary to go beyond the drift-diffusion approximation but to also include a more realistic contact model.

In the semiclassical framework, the BE provides the most precise information about the distribution of the charge carriers in the multidimensional phase space [19]. The most frequently applied method to solve the BE is the Monte-Carlo 
method [19], but there are no Monte-Carlo methods known to the authors that can perform small-signal simulations at complex frequencies required by the Dyakonov-Shur approach to plasma instabilities. On the other hand, deterministic solvers for the BE have been successfully used in the case of nanowire transistors (e.g., [20]), even including small-signal calculations [21]. In these cases, a transformation from the wavenumber to energy is used, which causes trouble in the case of a 1D $k$-space, because the density of states diverges at zero energy. This exacerbates the problems of small-signal calculations already encountered in the case of a 2D $k$-space [22]. Furthermore, in the ballistic limit the distribution function can be strongly asymmetric and discontinuous w.r.t. the wavenumber. This leads to further problems with the usual stabilization schemes based on the maximum entropy dissipation scheme [23] and the H-transformation [24]. These deficiencies are fatal in the numerical analysis of plasma resonances, where a numerically robust solver for the $\mathrm{BE}$ is required for handling the ballistic limit. In order to solve the above-mentioned problems, the BE is discretized directly in the phase space [16] and the quasiballistic limit of a nanowire transistor is investigated. The particular focus is on demonstrating a stabilization method, which is based on the method of characteristic curves and matrix exponentials. Thermal bath BCs, with the assumption of thermal equilibrium only for the electrons entering the device, give a more realistic description of the contacts [25]. The results are compared to the results of the DD equations.

The paper is organized as follows. Section 2 provides a description of the numerical framework used to evaluate the DC and small-signal behavior extending to the ballistic limit. In Sect. 3, the device structure is presented and the simulation results concentrating, in particular, on the small-signal drain self-admittance are discussed. Finally, in Sect. 4 the conclusions are drawn.

\section{Theory}

The numerical models are mostly constructed with the usual methods [16, 26-30], and only the new aspects are discussed. Since it is difficult to include the Pauli exclusion principle properly in moments-based transport models and it was neglected by Dyakonov and Shur, we will also neglect it in this investigation.

The device model for the silicon nanowire transistor consists of three partial differential equations, the Poisson, Schrödinger, and Boltzmann equations [20]. The Poisson equation is formulated for the $3 \mathrm{D}$ real space

$\nabla \cdot(\kappa \nabla \varphi)=q\left(n-N_{\mathrm{D}}\right)$, where $\kappa(\vec{r})$ is the permittivity at position $\vec{r}$ in real space, $\varphi(\vec{r}, t)$ the quasi-stationary potential at time $t, q$ the positive electron charge, $n(\vec{r}, t)$ the electron density and $N_{\mathrm{D}}(\vec{r})$ the donor concentration. The quasi-stationary approach is sufficient in this case, because even at $1 \mathrm{THz}$ the wave length of light in silicon is about $100 \mu \mathrm{m}$ and thus much larger than the device dimensions.

The device is sliced into $N_{\text {sec }}$ sections perpendicular to the transport direction ( $z$-direction), and the quasi-stationary Schrödinger equation is solved for $2 \mathrm{D}$ cross sections parallel to the $x y$-plane at fixed positions for $z$

$\frac{-\hbar^{2}}{2}\left(\frac{1}{m_{x x}^{\nu}} \frac{\partial^{2}}{\partial x^{2}}+\frac{1}{m_{y y}^{\nu}} \frac{\partial^{2}}{\partial y^{2}}\right) \psi^{\nu}-q \varphi \psi^{\nu}=\varepsilon_{\mathrm{sub}}^{\nu} \psi^{\nu}$

under the assumption that the wave functions vanish outside of the silicon region. $\hbar$ is the reduced Planck constant, $m_{x x}^{v}, m_{y y}^{v}$ the electron masses in $x$ and $y$ direction assuming a $\langle 100\rangle$ orientation of the silicon nanowire and parabolic bands, $\psi^{v}(\vec{r}, t)$ the envelope function and $\varepsilon_{\text {sub }}^{v}(z, t)$ the subband energy. The superscript $v=(v, s)$ contains the valley index $v$ of the minima of the conduction band and the subband index $s$. Again, the quasi-stationary approximation is used, because the largest part of the confining potential, which results in the boundary conditions, does not depend on time and due to the high symmetry of the device, which suppresses acoustic-wave-like plasma oscillations perpendicular to the transport direction.

The multi-subband 1D Boltzmann equation along the transport direction ( $z$-axis) is given by

$\frac{\partial f^{\nu}}{\partial t}+v^{\nu} \frac{\partial f^{\nu}}{\partial z}-\frac{1}{\hbar} \frac{\partial \varepsilon_{\mathrm{sub}}^{\nu}}{\partial z} \frac{\partial f^{\nu}}{\partial k}+S^{\nu}\{f\}=0$,

where $f^{v}(z, k, t)$ is the electron distribution function at wavenumber $k, v^{v}(k)$ the group velocity and $S\{f\}$ the scattering integral for single electron interactions [20]

$$
\begin{aligned}
S\{f\}= & \frac{L_{\text {sys }}}{2 \pi} \sum_{\nu^{\prime}} \int W^{v, v^{\prime}}\left(z, k, k^{\prime}, t\right) f^{\nu^{\prime}}\left(z, k^{\prime}, t\right) \\
& -W^{\nu^{\prime}, \nu}\left(z, k^{\prime}, k, t\right) f^{\nu}(z, k, t) \mathrm{d} k^{\prime} .
\end{aligned}
$$

$L_{\text {sys }}$ is the $1 \mathrm{D}$ system volume and $W^{\nu, v^{\prime}}\left(z, k, k^{\prime}, t\right)$ the rate of a transition from state $\left(k^{\prime}, v^{\prime}\right)$ into $(k, v)$ calculated by Fermi's golden rule [31]. Unless otherwise stated, we use the macroscopic relaxation time approximation (RTA) to improve the consistency of the BE and the moments-based models

$S\{f\} \approx \frac{\sum_{v^{\prime}} \int f^{\nu^{\prime}}\left(z, k^{\prime}, t\right) \frac{\mathrm{d} k^{\prime}}{\pi} p_{\mathrm{eq}}^{\nu}(k)-f^{\nu}(z, k, t)}{\tau_{\mathrm{RTA}}}$, 


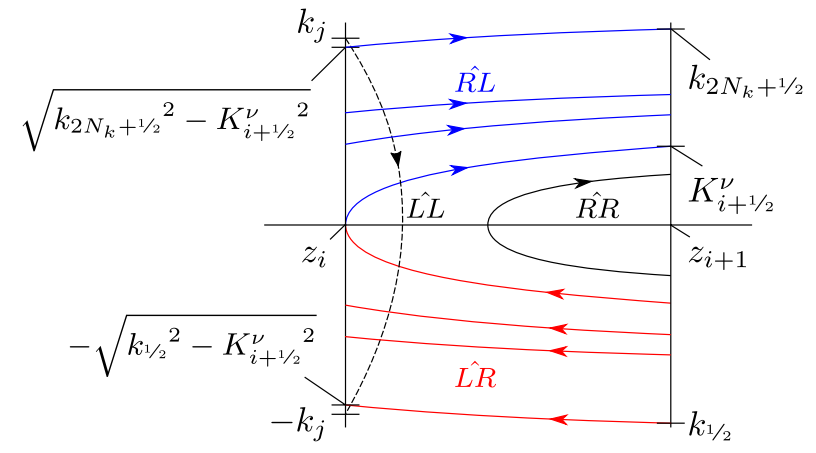

Fig. 1 Schematic representation of the characteristic curves for the case $\varepsilon_{\mathrm{sub}, i}^{\nu}>\varepsilon_{\mathrm{sub}, i+1}^{v}$

where $\tau_{\mathrm{RTA}}$ is the macroscopic relaxation time and $p_{\mathrm{eq}}^{v}(k)$ the one-particle Maxwell-Boltzmann distribution. The electron density is evaluated by

$n=\frac{2}{2 \pi} \sum_{v} \int f^{v} \mathrm{~d} k\left|\psi^{v}\right|^{2}$.

The Poisson and Schrödinger equations are discretized with the standard methods (finite volume method, dimensional splitting, finite differences etc. [26]). The resultant linear systems of equations are solved with PARDISO [32] and the eigenvalue problems with FEAST [33].

The $\mathrm{BE}$ is directly discretized in the phase space, which is the Cartesian product of the position along the channel axis $z$ and the axial wavevector $k$. An equidistant spatial grid $z_{i}=i \Delta z$ with $i=1, \ldots, N_{z}$ is introduced. In the $k$-space, a nonuniform grid with constant $\Delta k$ near $k=0$ and a $\Delta k$ at higher energies corresponding to a constant step in energy is chosen. The number of grid nodes for negative wavenumbers is $N_{k}$ and the same for positive ones. For the sake of legibility, the time arguments are dropped from now on.

We start our formulation by considering the ballistic limit $\left(W^{v, v^{\prime}}\left(z, k, k^{\prime}\right)=0\right)$, because this is the most critical case w.r.t. stability. Assuming a linear approximation for the subband energy $\varepsilon_{\text {sub }}^{v}(z)$ between the adjacent grid nodes $z_{i}$ and $z_{i+1}$, the force $F_{i}^{\nu}=-\left(\varepsilon_{\text {sub }, i+1}^{\nu}-\varepsilon_{\text {sub }, i}^{\nu}\right) /\left(z_{i+1}-z_{i}\right)$ is constant and the characteristic curves (ballistic electron path in the phase space) are parabolas. If the force is positive, the electrons are accelerated into the positive $z$-direction, and for $k>0$, an electron can move from $z_{i}$ to $z_{i+1}$ (blue trajectories in Fig. 1). The wavenumber of an electron in state $v$ starting at $z_{i}$ with $k\left(z_{i}\right)$ is at position $z_{i+1}$

$k\left(z_{i+1}\right)=\sqrt{k\left(z_{i}\right)^{2}+\left(K_{i+1 / 2}^{v}\right)^{2}}$

with
$K_{i+1 / 2}^{\nu}=\sqrt{\left|\frac{2 m_{z z}^{v}\left(\varepsilon_{\mathrm{sub}, i+1}^{v}-\varepsilon_{\mathrm{sub}, i}^{v}\right)}{\hbar^{2}}\right|}$.

$m_{z z}^{v}$ is the electron mass in transport direction. Thus, $k\left(z_{i+1}\right) \geq K_{i+1 / 2}^{v}$ holds. For electrons starting at $z_{i+1}$ and moving into the negative direction, we get

$k\left(z_{i}\right)=-\sqrt{k\left(z_{i+1}\right)^{2}-\left(K_{i+1 / 2}^{v}\right)^{2}}$.

The electrons can pass the barrier only, if their kinetic energy is larger than $\varepsilon_{\text {sub }, i}^{v}-\varepsilon_{\text {sub }, i+1}^{v}$ and thus $k\left(z_{i+1}\right) \leq-K_{i+1 / 2}^{v}$ (red trajectories in Fig. 1). If this is not the case $\left(-K_{i+1 / 2}^{v} \leq k\left(z_{i+1}\right) \leq 0\right)$, the electron returns to position $z_{i+1}$, where its wavenumber has reversed sign (black trajectories in Fig. 1).

The change in the wavenumber depends only on the difference in the subband energy and not on the exact force profile between both grid nodes. We can therefore assume that the subband energy is piecewise constant and changes abruptly at position $z_{i+1 / 2}=\left(z_{i+1}+z_{i}\right) / 2$. Due to the Liouville theorem, the electron flux at position $z_{i+1 / 2}$ on the lefthand side of the step must equal the one on the right-hand side. For $k_{\mathrm{L}}>0$, where the superscript $\mathrm{L}$ indicates quantities on the LHS and R the ones on the RHS, we get

$v^{v}\left(k_{\mathrm{R}}\right) f_{\mathrm{R}}^{v}\left(z_{i+1 / 2}, k_{\mathrm{R}}\right) \mathrm{d} k_{\mathrm{R}}=v^{v}\left(k_{\mathrm{L}}\right) f_{\mathrm{L}}^{v}\left(z_{i+1 / 2}, k_{\mathrm{L}}\right) \mathrm{d} k_{\mathrm{L}}$

with

$k_{\mathrm{R}}=\sqrt{k_{\mathrm{L}}^{2}+\left(K_{i+1 / 2}^{\nu}\right)^{2}}$.

Thus, for the piecewise constant subband energy profile the characteristic curves are no longer continuous at $z_{i+1 / 2}$ and the values of the distribution function on the left and right sides of the step have to be distinguished. For $k_{\mathrm{R}}<-K_{i+1 / 2}^{v}$, the electrons can pass the barrier from right to left resulting in the negative root for the wavenumber

$k_{\mathrm{L}}=-\sqrt{k_{\mathrm{R}}^{2}-\left(K_{i+1 / 2}^{v}\right)^{2}}$.

together with (10). For $\left|k_{\mathrm{R}}\right|<K_{i+1 / 2}^{v}$, we get reflection

$f_{\mathrm{R}}^{v}\left(z_{i+1 / 2}, k_{\mathrm{R}}\right)=f_{\mathrm{R}}^{\nu}\left(z_{i+1 / 2},-k_{\mathrm{R}}\right)$.

Discrete equations are obtained by box integration in the $k$-space. The $k$-grid has $2 N_{k}$ grid nodes, which are symmetrically distributed around zero and the value zero itself is excluded from the grid. The $j$ th box is given by $k_{j}^{-}<k<k_{j}^{+}$ with its boundaries $k_{j}^{ \pm}=\left(k_{j}+k_{j \pm 1}\right) / 2$ and $j=1, \ldots, 2 N_{k}$. The interpolation function for the $j$ th box is one inside the $j$ th box and zero outside 
$\Omega_{j}(k)=\Theta\left(\frac{k_{j}^{+}-k_{j}^{-}}{2}-\left|k-\frac{k_{j}^{+}+k_{j}^{-}}{2}\right|\right)$,

where $\Theta(x)$ is the Heaviside function. The distribution functions are assumed to be piecewise constant with the value $f^{v, j}(z)$ for the $j$ th box

$f^{\nu}(z, k) \approx \sum_{j=1}^{2 N_{k}} f^{\nu, j}(z) \Omega_{j}(k)$.

On the LHS/RHS of the step in subband energy, we have

$f_{\mathrm{L}, i+1 / 2}^{\nu, j}=f_{\mathrm{L}}^{\nu, j}\left(z_{i+1 / 2}\right), \quad f_{\mathrm{R}, i+1 / 2}^{\nu, j}=f_{\mathrm{R}}^{\nu, j}\left(z_{i+1 / 2}\right)$.

Particle flux conservation is mapped onto the discrete $k$-grid by box integration. This allows to discretize (10) together with (13) for $j>N_{k}$ (positive wavenumbers)

$$
\begin{aligned}
f_{\mathrm{R}, i+1 / 2}^{v, j} & \int_{k_{j}^{-}}^{k_{j}^{+}} v^{v}\left(k_{\mathrm{R}}\right) \mathrm{d} k_{\mathrm{R}} \\
= & \sum_{j^{\prime}=N_{k}+1}^{2 N_{k}} f_{\mathrm{L}, i+1 / 2}^{v, j^{\prime}} \int_{\mathrm{K}_{\min }^{v, j}}^{\mathrm{K}_{\text {max }}^{v j}} v^{\nu}\left(k_{\mathrm{L}}\right) \Omega_{j^{\prime}}\left(k_{\mathrm{L}}\right) \mathrm{d} k_{\mathrm{L}} \\
& +f_{\mathrm{R}, i+1 / 2}^{v, 2 N_{k}-j+1} \int_{k_{j}^{-}}^{k_{j}^{+}} v^{\nu}\left(k_{\mathrm{R}}\right) \Theta\left(K_{i+1 / 2}^{v}-k_{\mathrm{R}}\right) \mathrm{d} k_{\mathrm{R}} .
\end{aligned}
$$

The first term on the RHS describes the flux of electrons from the left to the right and the second term electrons reflected at the step. The integration limits are defined as

$\mathrm{K}_{\min }^{v, j}=\sqrt{\max \left\{k_{j-1 / 2}^{2}-\left(K_{i+1 / 2}^{v}\right)^{2}, 0\right\}}$,

$\mathrm{K}_{\max }^{v j}=\sqrt{\max \left\{k_{j+1 / 2}^{2}-\left(K_{i+1 / 2}^{v}\right)^{2}, 0\right\}}$.

If we assemble the $N_{k}$ values of the distribution function for positive wavenumbers into a vector on the LHS of the step $\vec{f}_{\mathrm{Lp}, \mathrm{i}+1 / 2}^{v}$, on the RHS $\vec{f}_{\mathrm{Rp}, \mathrm{i}+1 / 2}^{v}$ and for negative wavenumbers $\vec{f}_{\mathrm{Ln}, \mathrm{i}+1 / 2}^{v}, \vec{f}_{\mathrm{Rn}, \mathrm{i}+1 / 2}^{v}$, we can formulate (17) with matrices

$\hat{D}_{\mathrm{p}}^{v} \vec{f}_{\mathrm{Rp}, \mathrm{i}+1 / 2}^{v}=\hat{R} \hat{L}_{i+1 / 2}^{v} \vec{f}_{\mathrm{Lp}, \mathrm{i}+1 / 2}^{v}+\hat{R R} \hat{i+1 / 2}_{\mathrm{Rn}, \mathrm{i}+1 / 2}^{v} \vec{f}^{v}$,

where the diagonal $N_{k} \times N_{k}$ matrix $\hat{D}_{\mathrm{p}}^{v}$ is invertible and does not depend on the position in real space

$\left[\hat{D}_{\mathrm{p}}^{v}\right]_{j j^{\prime}}=\int_{k_{j+N_{k}}^{-}}^{k_{j+N_{k}}^{+}} v^{v}(k) \mathrm{d} k \delta_{j, j^{\prime}}$

for $j, j^{\prime}=1, \ldots, N_{k}$. The other two matrices are
$\left[\hat{R} L_{i+1 / 2}^{v}\right]_{j j^{\prime}}=\int_{\mathrm{K}_{\min }^{v, j+N_{k}}}^{\mathrm{K}_{\max }^{v j+N_{k}}} v^{v}(k) \Omega_{j^{\prime}+N_{k}}(k) \mathrm{d} k$

and

$\left[\hat{R R}_{i+1 / 2}^{v}\right]_{j j^{\prime}}=\int_{k_{j+N_{k}}^{-}}^{k_{j+N_{k}}^{+}} v^{v}(k) \Theta\left(K_{i+1 / 2}^{v}-k\right) \mathrm{d} k \delta_{j^{\prime}, N_{k}-j+1}$.

Flux conservation for particles moving from right to left results with (10) and (12) in

$\hat{D}_{\mathrm{n}}^{v} \vec{f}_{\mathrm{Ln}, i+1 / 2}^{v}=\hat{L} \hat{R}_{i+1 / 2}^{v} \vec{f}_{\mathrm{Rn}, i+1 / 2}^{v}+\hat{L} \hat{L}_{i+1 / 2}^{v} \vec{f}_{\mathrm{Lp}, i+1 / 2}^{v}$.

The second term on the RHS is due to the assumption of a maximal positive wavenumber $k_{2 N_{k}}^{+}$, and all particles, which were to exceed this value while moving from left to right $\left(\sqrt{k_{\mathrm{L}}^{2}+\left(K_{i+1 / 2}^{v}\right)^{2}}>k_{2 N_{k}}^{+}\right)$, are instead reflected to ensure flux conservation (dashed black line in Fig. 1). Furthermore, a similar set of equations can be formulated for a positive step in energy.

Under stationary conditions, the ballistic distribution function does not depend on position for a constant subband energy and within a box of the real space grid $z_{i-1 / 2}<z<z_{i+1 / 2}$ we get

$\vec{f}_{\mathrm{Lp}}^{v}\left(z_{i+1 / 2}\right)=\vec{f}_{\mathrm{Rp}}^{v}\left(z_{i-1 / 2}\right)=\vec{f}_{\mathrm{p}, i}^{v}$

$\vec{f}_{\mathrm{Ln}}^{v}\left(z_{i+1 / 2}\right)=\vec{f}_{\mathrm{Rn}}^{v}\left(z_{i-1 / 2}\right)=\vec{f}_{\mathrm{n}, i}^{v}$.

Due to the TB-BCs, the distribution function of the incoming particles is given by the equilibrium one $\vec{f}_{\text {eq }}^{v}$ [25]

$\vec{f}_{\mathrm{p}, 1}^{v}=\vec{f}_{\mathrm{p}, \mathrm{eq}, \mathrm{SRC}}^{v}$

$\vec{f}_{\mathrm{n}, N_{z}}^{v}=\vec{f}_{\mathrm{n}, \mathrm{eq}, \mathrm{DRN}}^{v}$.

The Fermi energies of the contacts are chosen such that the space charge densities are zero within the contacts at equilibrium. This closes the system of equations, and in most cases, a unique solution can be obtained for $\overrightarrow{\mathrm{p}}_{\mathrm{p}, i}^{v}, \vec{f}_{\mathrm{n}, i}^{v}{ }^{1}$ Since the Pauli exclusion principle occurs in the BE only in the scattering integral, it can be included in the ballistic case by using Fermi functions instead of Boltzmann distributions in the boundary conditions.

\footnotetext{
$\overline{1}$ For certain profiles of subband energy with minima within the device, some parts of the phase space might be decoupled from the rest due to the lack of scattering and the distribution function in those parts is undetermined [34].
} 
The solution becomes unique for arbitrary subband profiles, if scattering is taken into account, because scattering couples all states for a given position [20]. Since scattering couples different subbands and valleys, the index $v$ is dropped and the vector of the distribution function contains from now on the distribution functions for all values of $v$. Due to the assumption of a piecewise constant subband energy, the force is zero within a box of the real space. Integration of the $\mathrm{BE}$ over a box in $k$-space yields

$$
\underbrace{\left(\begin{array}{cc}
\hat{D}_{\mathrm{p}} & \\
& \hat{D}_{\mathrm{n}}
\end{array}\right)}_{=\hat{D}_{v}} \frac{\partial}{\partial z} \underbrace{\left(\begin{array}{l}
\vec{f}_{\mathrm{p}}(z) \\
\vec{f}_{\mathrm{n}}(z)
\end{array}\right)}_{=\vec{f}(z)}+\underbrace{\left(\begin{array}{cc}
\hat{S}_{\mathrm{pp}} & \hat{S}_{\mathrm{pn}} \\
\hat{S}_{\mathrm{np}} & \hat{S}_{\mathrm{nn}}
\end{array}\right)}_{=\hat{S}(z)}\left(\begin{array}{l}
\vec{f}_{\mathrm{p}}(z) \\
\vec{f}_{\mathrm{n}}(z)
\end{array}\right)=\overrightarrow{0}
$$

with the scattering integral

$$
\begin{aligned}
{[\hat{S}(z)]_{j j^{\prime}}^{v, v^{\prime}} } & =\frac{L_{\text {sys }}}{2 \pi} \int_{k_{j}^{-}}^{k_{j}^{+}}\left(\int_{k_{j^{\prime}}}^{k_{j^{\prime}}^{+}} W^{\nu, \nu^{\prime}}\left(z, k, k^{\prime}\right) \mathrm{d} k^{\prime}\right. \\
& \left.-\sum_{\nu^{\prime \prime}} \int_{\infty}^{\infty} W^{\nu^{\prime \prime}, v}\left(z, k^{\prime \prime}, k\right) \mathrm{d} k^{\prime \prime} \delta_{\nu, v^{\prime}} \delta_{j, j^{\prime}}\right) \mathrm{d} k .
\end{aligned}
$$

Due to scattering, the solution depends on the $z$-coordinate within a box of the real space, even if we assume that the transition rate itself does not depend on position within the box. With $\vec{f}_{i}=\vec{f}\left(z_{i}\right), \hat{S}_{i}=\hat{S}\left(z_{i}\right)$ and the matrix exponential [35], the solution is within the $i$ th box of the real space grid

$\vec{f}(z)=\exp \left(\left(z_{i}-z\right) \hat{D}_{v}^{-1} \hat{S}_{i}\right) \vec{f}_{i}$.

With the abbreviation $\hat{C}_{i}(z)=\left(z_{i}-z\right) \hat{D}_{v}^{-1} \hat{S}_{i}$, the solution at the boundaries of the $i$ th box in real space is

$\vec{f}_{\mathrm{L}, i+1 / 2}=\exp \left(\hat{C}_{i}\left(z_{i+1 / 2}\right)\right) \vec{f}_{i}$

$\vec{f}_{\mathrm{R}, i-1 / 2}=\exp \left(\hat{C}_{i}\left(z_{i-1 / 2}\right)\right) \vec{f}_{i}$.

These equations replace (25), (26) of the ballistic case. Thus, it is possible to calculate the distribution function on the leftand right-hand side of the steps based on the distribution function on the $i$ th grid node and a complete set of equations can again be assembled. The matrix exponential can be calculated with the methods described in Ref. [35], but for large matrices the numerical accuracy might not be sufficient. In this case, Expokit together with an iterative solver, which requires only multiplications of the matrix exponential with a vector, yields results with a higher precision and is more CPU efficient [36]. If the Pauli exclusion principle were included in the scattering integral, (29) would become nonlinear and could be integrated by ODE-solvers.
For small-signal analysis, we assume a time dependence for the solution quantities given by $x(t)=x+\mathfrak{R}\{\delta x \exp (s t)\}$, where $x$ is the large-signal stationary solution, $\delta x$ the complex phasor of the small-signal solution and $s=\sigma+\mathrm{i} \omega$ the complex frequency, where $\sigma$ is the real part describing a damped $(\sigma<0)$ or growing $(\sigma>0)$ solution and $\omega=2 \pi f$ the imaginary part corresponding to a harmonic oscillation with the frequency $f$. The time derivative in the $\mathrm{BE}$ can therefore be replaced by $s$, and within a box of the spatial grid, the linearized $\mathrm{BE}$ has two additional terms compared to the stationary case (29)

$s \hat{D}_{k} \delta \vec{f}(z)+\hat{D}_{v} \frac{\partial \delta \vec{f}(z)}{\partial z}+\hat{S} \delta \vec{f}(z)=-\delta \hat{S} \vec{f}(z)$

$\hat{D}_{k}$ is a diagonal matrix, which is due to box integration in $k$-space and contains the $k$-space box volumes

$\left[\hat{D}_{k}\right]_{j j^{\prime}}^{\nu v^{\prime}}=\left(k_{j}^{+}-k_{j}^{-}\right) \delta_{v, v^{\prime}} \delta_{j, j^{\prime}}$.

Since the scattering integral depends on the subband energies and wave functions, linearization of the scattering integral yields the term on the RHS of (34). The corresponding derivatives are calculated by the chain rule and stationary perturbation theory for the SE [30]. With the abbreviation $\hat{C}_{i}^{\prime}(z)=\left(z_{i}-z\right) \hat{D}_{v}^{-1}\left(\hat{S}_{i}+s \hat{D}_{k}\right)$, the solution of the small-signal BE is obtained

$$
\begin{aligned}
\delta \vec{f}(z)= & \exp \left(\hat{C}_{i}^{\prime}(z)\right) \delta \vec{f}_{i} \\
& +\int_{0}^{1} \exp \left(\hat{C}_{i}^{\prime}(z)(1-\alpha)\right) \delta \hat{C}_{i}(z) \exp \left(\hat{C}_{i}(z) \alpha\right) \mathrm{d} \alpha \vec{f}_{i} .
\end{aligned}
$$

With this result, the values of the small-signal distribution function at the boundaries of the real-space box can be calculated by numerical means. Linearization of (20) yields

$$
\begin{aligned}
\hat{D}_{\mathrm{p}}^{v} \delta \vec{f}_{\mathrm{Rp}, \mathrm{i}+1 / 2}^{v}= & \hat{R L}_{i+1 / 2}^{v} \delta \vec{f}_{\mathrm{Lp}, \mathrm{i}+1 / 2}^{v}+\delta \hat{R L}_{i+1 / 2}^{v} \vec{f}_{\mathrm{Lp}, \mathrm{i}+1 / 2}^{v} \\
& +\hat{R R}_{i+1 / 2}^{v} \delta \vec{f}_{\mathrm{Rn}, \mathrm{i}+1 / 2}^{v}+\delta \hat{R R_{i+1 / 2}^{v}} \overrightarrow{\mathrm{fn}}_{\mathrm{Rn}, \mathrm{i}+1 / 2}^{v}
\end{aligned}
$$

and (24) similarly. These equations together with the smallsignal PE and the small-signal version of the boundary conditions (27), (28)

$\left\langle\vec{f}_{\mathrm{p}, 1}^{v}=\overrightarrow{0}, \quad \delta \vec{f}_{\mathrm{n}, N_{z}}^{v}=\overrightarrow{0}\right.$

can be used to assemble a linear system of equations for the complete system. For the calculation of the small-signal terminal currents, a formulation of the Ramo-Shockley theorem is used that is consistent with the presented numerical framework [21, 37]. Thus, admittance parameters can be calculated as a function of the complex frequency $s$, as required by the analysis of plasma instabilities. 


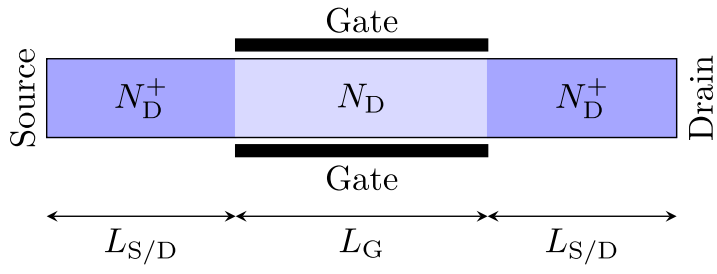

Fig. 2 Schematic representation of the nanowire NMOSFET. The carriers are confined in the $x-y$ plane and the transport happens along the $z$-axis from source to drain

Table 1 Parameters of the nanowire NMOSFET

\begin{tabular}{ll}
\hline Parameter & Value \\
\hline Oxide thickness $\left(t_{\mathrm{ox}}\right)$ & $1 \mathrm{~nm}$ \\
Nanowire area & $5 \mathrm{~nm} \times 4 \mathrm{~nm}$ \\
Gate oxide material & $\mathrm{SiO}_{2}\left(\kappa_{\mathrm{ox}}=3.9\right)$ \\
$N_{\mathrm{D}}^{+}$ & $5 \times 10^{19} \mathrm{~cm}^{-3}$ \\
$N_{\mathrm{D}}$ & $1 \times 10^{18} \mathrm{~cm}^{-3}$ \\
$L_{\mathrm{S} / \mathrm{D}}, L_{\mathrm{G}}$ & $20 \mathrm{~nm}, 20 \mathrm{~nm}$ \\
\hline
\end{tabular}

\section{Results and discussion}

The test structure with a gate all-around architecture is shown in Fig. 2. The rectangular channel cross section is $5 \mathrm{~nm} \times 4 \mathrm{~nm}$ with a gate oxide thickness of $1 \mathrm{~nm}$ (further details are given in Table 1).

First, simulation of the ballistic case is demonstrated, because this is the numerically most challenging case. Since scattering is neglected, particles cannot transfer between valleys and subbands, and only a single parabolic subband with $m_{x x}=m_{z z}=0.19 m_{\mathrm{e}}, m_{y y}=0.98 m_{\mathrm{e}}$ is simulated ( $m_{\mathrm{e}}$ is the electron rest mass). The $k$-space grid has a constant spacing of $\Delta k=0.0225 \mathrm{~nm}^{-1}$ at low energies and at higher ones a spacing corresponding to an energy of $2.5 \mathrm{meV}$. The maximal energy is $1.01 \mathrm{eV}$. The structure described in Table 1 is simulated with a grid spacing in real space of $0.5 \mathrm{~nm}$, and the doping profile is shown in Fig. 3. To demonstrate the stability of the approach, the electron density in the device is shown for different gate and drain voltages under ballistic conditions (Fig. 3). The electron density can vary by more than 10 orders of magnitude without any problems. In Fig. 4, distribution functions in the $k$-space are shown near the drain and the source/channel barrier (top of the subband energy). The distribution function is positive in the whole phase space and shows no spurious oscillations. Due to the larger number of electrons moving from the left to the right than into the other direction, the distribution function is strongly asymmetric. Since the electrons do not loose energy by

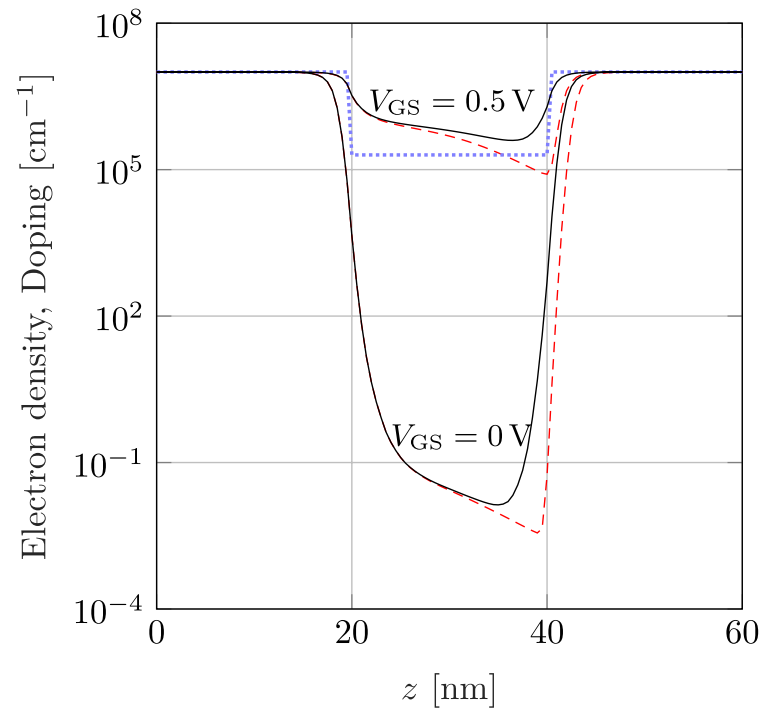

Fig. 3 Doping profile (dotted blue curve) and electron density at $V_{\mathrm{DS}}=0.1 \mathrm{~V}$ (solid curves) and $V_{\mathrm{DS}}=0.5 \mathrm{~V}$ (dashed curves) and room temperature (Color figure online)

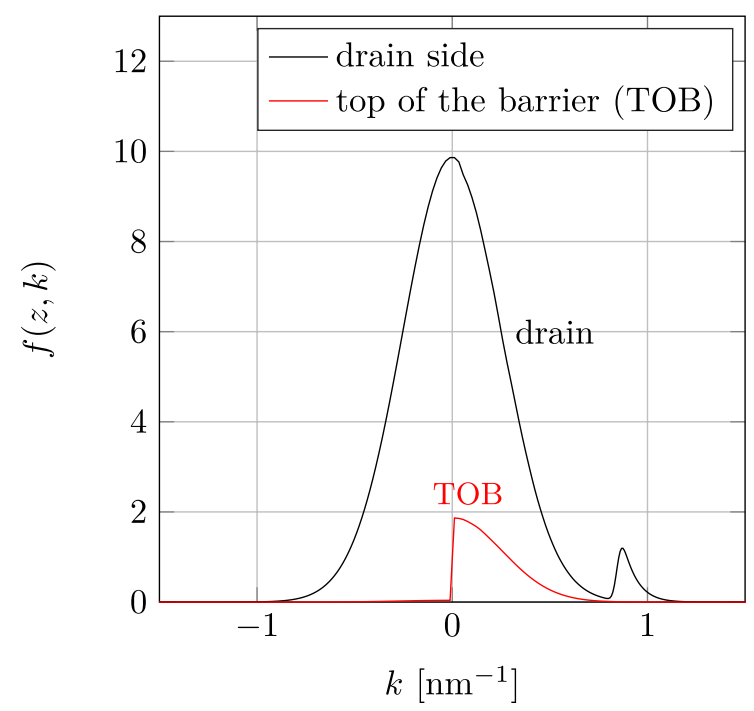

Fig. 4 Distribution functions near the drain contact and top of the barrier for the ballistic case at $V_{\mathrm{GS}}=0.5 \mathrm{~V}, V_{\mathrm{DS}}=0.1 \mathrm{~V}$ and room temperature

scattering when moving from source to drain, they gain $q V_{\mathrm{DS}}=100 \mathrm{meV}$ in energy and a second maximum occurs in the distribution function near the drain at positive wavenumbers. Although the cutoff by the source/channel barrier is abrupt, the left flank of the second maximum near the drain is not as abrupt as at the top of the barrier. This is due to the finite $k$-space grid, which leads to artificial diffusion in the $k$-space, because a $k$-space box on the LHS of a step of the subband energy in real space is connected to multiple boxes on the RHS and vice versa (see (17)). 


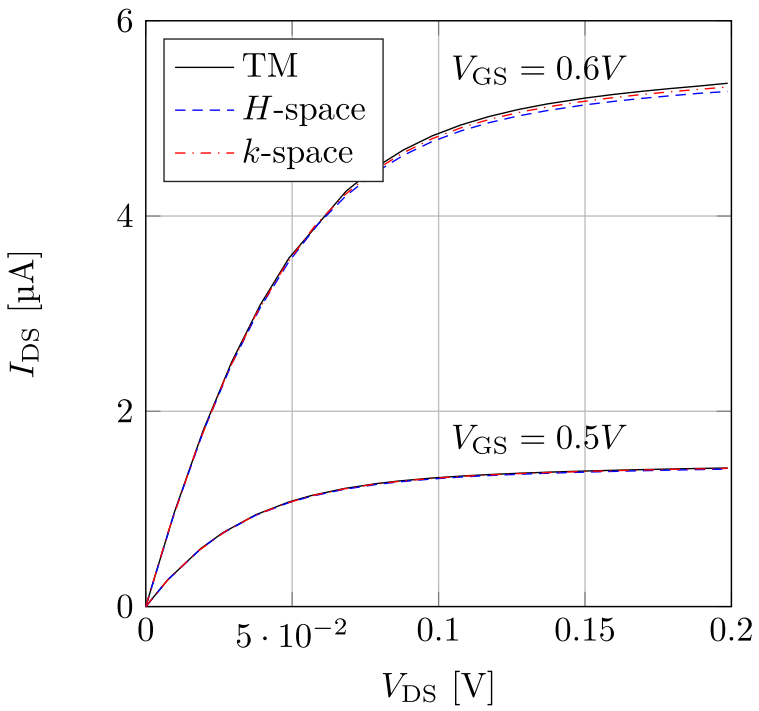

Fig. 5 Calculated $I_{\mathrm{DS}}-V_{\mathrm{DS}}$ curves of the silicon $\mathrm{N}^{+} \mathrm{NN}^{+}$transistor at different gate voltages. The results are obtained from simulation of the $\mathrm{BE}$ under $H$-transformation, simulation of the $\mathrm{BE}$ in phase space, and tenth-order moments-based equations (TM)

This is a fundamental problem of tensor-product grids in phase space and occurs regardless of the treatment of the derivative in $k$-space, because any formulation of the derivative in $k$-space involves multiple grid nodes. In principle, a finer grid in $k$-space could reduce the diffusion, but a finer $k$-space grid requires a finer real space grid to prevent numerical problems and a certain level of diffusion cannot be avoided. On the other hand, the impact of the artificial diffusion is weak, the phase-space solver based on the tensor-product grid is stable in the ballistic case, and the problems due to the H-transformation in the case of the small-signal parameters are avoided.

In silicon nanowires, the mobility is rather low due to strong scattering resulting in diffusive transport [34], and it is not clear whether the numerical methods developed for the ballistic case will work. To investigate this, a silicon nanowire is simulated. The electron bandstructure in silicon is modeled in the conventional way accounting for six ellipsoidal and parabolic valleys, and five subbands are considered in each valley. As for the scattering mechanisms, various inter-valley and intra-valley electron-phonon interactions are included according to [38] and the deformation potential of elastic phonon scattering is adjusted in highdoped regions to reproduce the results of [20]. The simulations are performed at room temperature, assuming a $\langle 100\rangle$ orientation in transport direction. The calculated $I_{\mathrm{DS}}-V_{\mathrm{DS}}$ curves are presented in Fig. 5 (red curves), and the results are smooth.This shows that also strongly diffusive transport can be simulated. In order to validate the model, the results are compared to consistent simulations by other

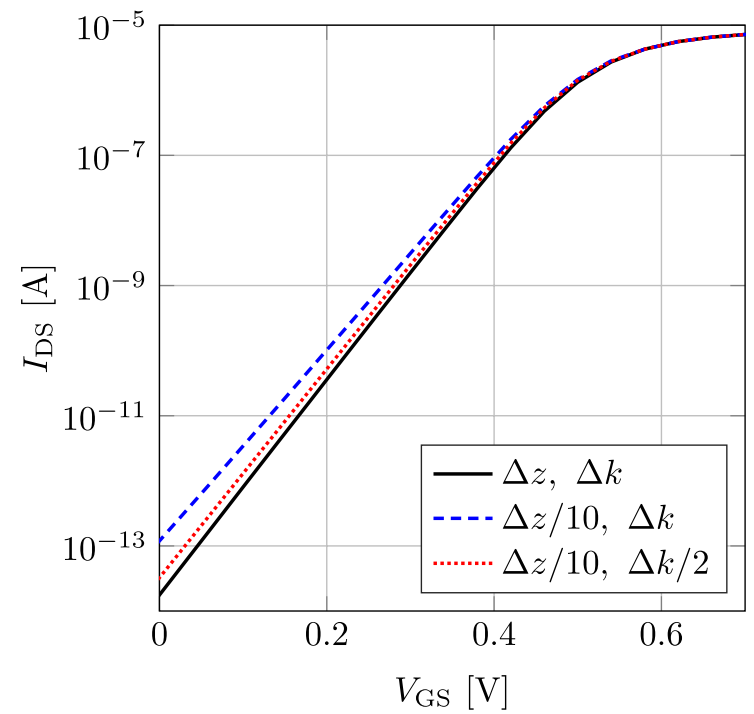

Fig. 6 Calculated $I_{\mathrm{DS}}-V_{\mathrm{GS}}$ curves of the silicon $\mathrm{N}^{+} \mathrm{NN}^{+}$transistor, obtained from simulation of the BE in phase space. $\Delta z=2 \mathrm{~nm}$ and $\Delta k=0.055 \mathrm{~nm}^{-1}$ were chosen for these simulations

means. The BE is solved in addition based on the $H$-transformation [21] and by projection onto the first 10 Hermitian polynomials (TM) [14], and the results are almost identical. At $V_{\mathrm{DS}}=0.2 \mathrm{~V}$, the calculations by the $H$-transformed $\mathrm{BE}$ and moments equations differ by $\approx 1.5 \%$ for $V_{\mathrm{GS}}=0.6 \mathrm{~V}$ and $\approx 0.8 \%$ for $V_{\mathrm{GS}}=0.5 \mathrm{~V}$, where an energy grid with a uniform spacing of approximately $2.6 \mathrm{meV}$ has been used for the $H$-transformed BE. Figure 6 shows the $I_{\mathrm{DS}}-V_{\mathrm{GS}}$ characteristics of the device for $V_{\mathrm{DS}}=0.1 \mathrm{~V}$ and different discretization parameters. The artificial diffusion in phase space translates to artificial carrier heating and increases the subthreshold slope of the device. This effect, however, is not very significant, and our simulations show that even for a coarse $k$-grid $\left(\Delta k=0.055 \mathrm{~nm}^{-1}\right)$, the subthreshold slope changes from $\mathrm{SS}=60.5 \mathrm{mV} / \mathrm{dec}$ to $\mathrm{SS}=66.5 \mathrm{mV} / \mathrm{dec}$ as we move from a very coarse spatial grid $(\Delta z=2 \mathrm{~nm})$ to a very fine one $(\Delta z=0.2 \mathrm{~nm})$. Refining the $k$-grid alleviates this problem and we have $\mathrm{SS}=62.5 \mathrm{mV} / \mathrm{dec}$ for $\Delta k=0.0225 \mathrm{~nm}^{-1}$. Although $\Delta k$ cannot be reduced below a certain value for a given $\Delta z$, the spurious increase in the subthreshold slope can be reduced to such a level that it is negligible (at least at room temperature). Keeping this in mind, our results confirm the validity of all three approaches for the simulation of conventional devices with relatively low mobilities.

Next, we investigate the quasi-ballistic limit of the nanowire transistor. In the rest of this paper, single-subband and single-valley transport together with the RTA for the scattering integral is assumed for the sake of CPU efficiency and consistency of the BE and the momentsbased models. In the case of high mobilities, the impact of 


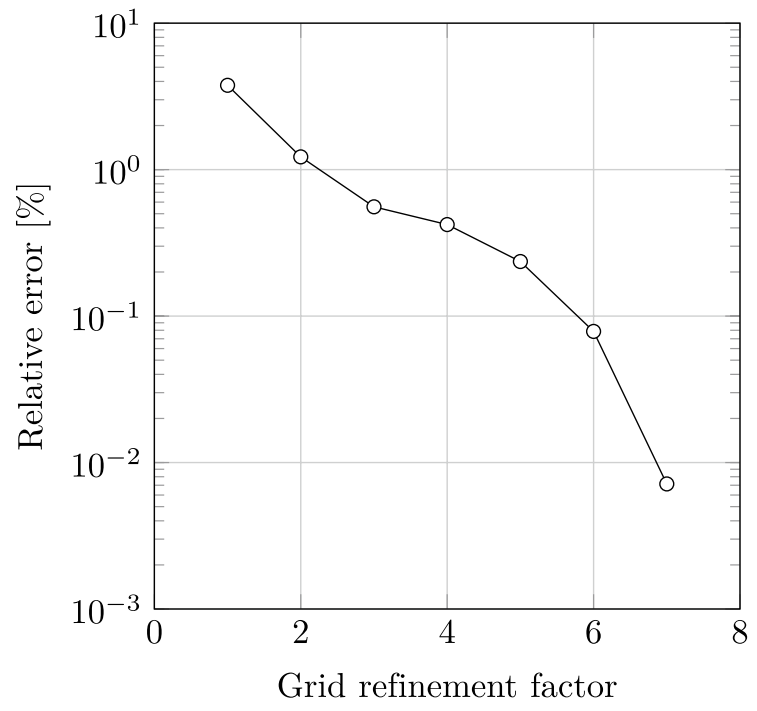

Fig. 7 Convergence of $\underline{Y}_{\mathrm{DD}}$ at zero frequency. Ballistic simulations for $L_{\mathrm{G}}=20 \mathrm{~nm} . V_{\mathrm{GS}}=0.6 \mathrm{~V}, V_{\mathrm{DS}}=0 \mathrm{~V}$

scattering is weak anyway and the error due to the RTA is small for small $V_{\mathrm{DS}}$. In order to check the accuracy of our BE solver, the ballistic small-signal drain self-admittance $\underline{Y}_{\mathrm{DD}}$ is calculated for different grid refinement factors and the relative error w.r.t. the finest grid is shown in Fig. 7. It is important to note that the refinements of the $z$-and $k$-grids have contrary impacts on the numerical stability of the discretized equations, which leads to a Courant-Friedrich-Lewy-like condition for $\Delta z$ and $\Delta k$. While the $k$-grid should be fine enough to capture discontinuities, the $z$-grid must be refined accordingly to prevent numerical instabilities. Hence, Fig. 7 presents the relative error with simultaneous refinement of both $z$-and $k$-grids. The error decreases for finer grids and the method converges.

We investigate the drain self-admittance as a function of the frequency, because it is an important quantity to determine the impact of plasma waves on the device behavior. For example, a negative real part of the drain self-admittance corresponds to an instability and could enable the generation of $\mathrm{THz}$ waves [7,39]. Results of the $\mathrm{BE}$ are compared to the ones calculated with projections onto different numbers of Hermitian polynomials and boundary conditions, where the first two Hermitian polynomials result in the DD model. In order to obtain a plasma instability, specific bias conditions have to be applied to the contacts of the device. The gate/source port should be short-circuited and the drain/source port open (i.e., the drain self-admittance should be zero). These bias conditions should not be confused with the boundary conditions of the transport models at the contacts inside the device, which are determined by the contact model.

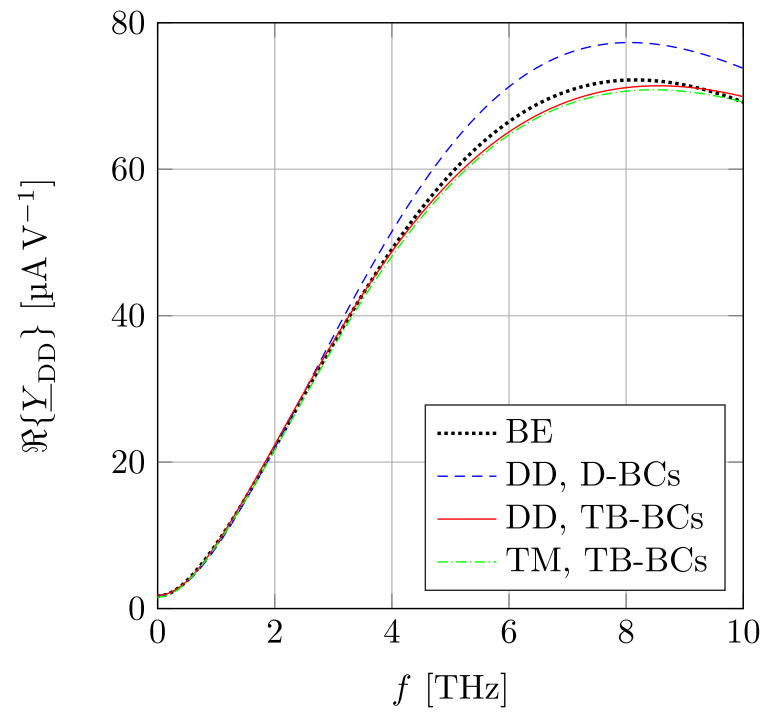

Fig. $8 \Re\left\{\underline{Y}_{\mathrm{DD}}(\mathrm{i} 2 \pi f)\right\}$ versus frequency at $V_{\mathrm{GS}}=0.5 \mathrm{~V}$ and $V_{\mathrm{DS}}=0 \mathrm{~V}$. Simulations are performed for the mobility of $\mu=100 \mathrm{~cm}^{2} / \mathrm{Vs}$

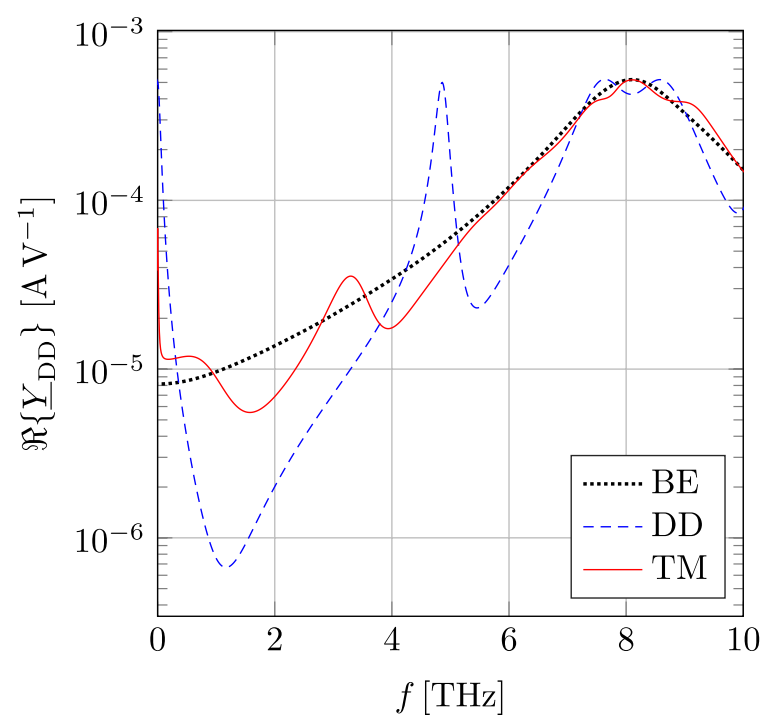

Fig. $9 \Re\left\{\underline{Y}_{\mathrm{DD}}\right\}$ versus frequency at $V_{\mathrm{GS}}=0.5 \mathrm{~V}$ and $V_{\mathrm{DS}}=0 \mathrm{~V}$. Simulations are performed for a mobility of $\mu=10^{6} \mathrm{~cm}^{2} /$ Vs and TB-BCs are applied

Results are presented for mobilities of $\mu=q \tau_{\mathrm{RTA}}^{\nu} / m_{z z}^{\nu}=100 \mathrm{~cm}^{2} / \mathrm{Vs}$ in Fig. 8 (strong damping of plasma waves) and $\mu=10^{6} \mathrm{~cm}^{2} / \mathrm{Vs}$ (quasi-ballistic case) in Fig. 9 for TB-BCs and 10 for D-BCs. For lower mobilities, good agreement between the two methods is observed over the entire frequency range and the details of the source and drain boundary conditions do not play an important role, i.e., even the drift-diffusion (DD) model with D-BCs can give reasonably accurate predictions in the diffusive regime for frequencies $f<3 \mathrm{THz}$. Although the DD model deviates 


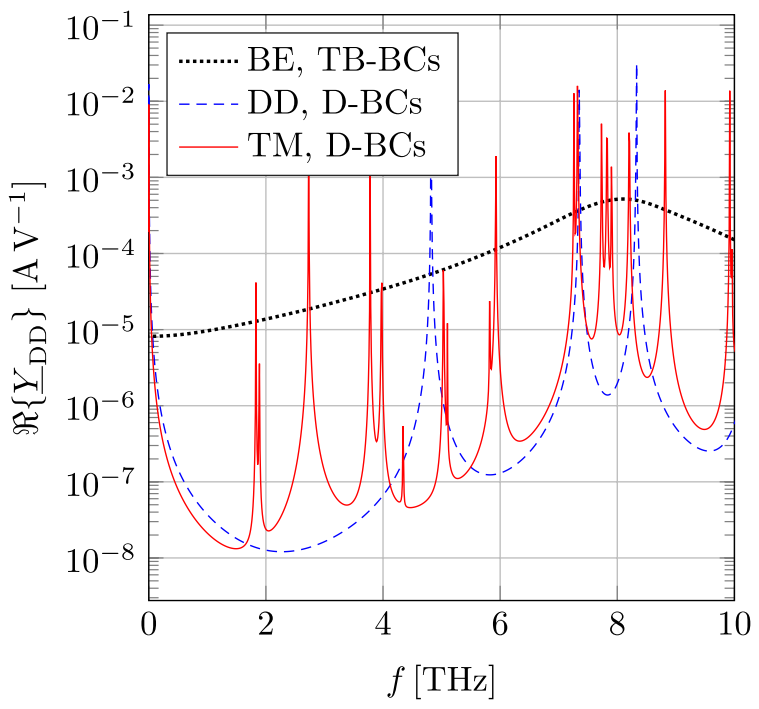

Fig. $10 \Re\left\{\underline{Y}_{\mathrm{DD}}\right\}$ versus frequency at $V_{\mathrm{GS}}=0.5 \mathrm{~V}$ and $V_{\mathrm{DS}}=0 \mathrm{~V}$. Simulations are performed for a mobility of $\mu=10^{6} \mathrm{~cm}^{2} / \mathrm{Vs}$ and D-BCs are applied (BE with TB-BCs)

from the results of the BE solver at higher frequencies (e.g., it has an error of $\approx 8 \%$ at $f=8 \mathrm{THz}$ ), it performs well in predicting the overall behavior of the real part. Truncation of the Hermite polynomial expansion at higher orders and TB-BCs improve the accuracy of the results.

However, when the mobility is increased to $\mu=10^{6} \mathrm{~cm}^{2} / \mathrm{Vs}$, moments of the BE fail to even provide a qualitative description of the device's small-signal behavior (Fig. 9). Although the model with ten moments (TM) does a better job than the DD model, the overall error below $5 \mathrm{THz}$ is rather large. The DD model not only shows an artificial peak at about $5 \mathrm{THz}$, it also yields a far too large admittance at zero frequency. This is a well-known deficiency of the DD model [40, 41]. The situation becomes worse, if we change the boundary conditions of the moments-based models from TB-BCs to D-BC, which were used by Dyakonov and Shur (Fig. 10). The unrealistically large values of $\Re\{\underline{Y}\}$ at zero frequency increase by another $2-3$ orders of magnitude. In addition, the peaks of the real part of the admittance become much sharper and higher and the minima smaller. The latter result is important, because the Dyakonov-Shur instability corresponds to a zero of the drain self-admittance (pole of the drain self-impedance for a short-circuited input). In Fig. 11, the absolute value of the drain self-admittance is shown for the DD model with different $\mathrm{BCs}$, where the maxima and minima correspond to the poles and zeros, respectively. For D-BCs, the poles and zeros are lined up at $\sigma=-1 / 2 \tau_{\mathrm{RTA}}$ [6], which is very small for $\mu=10^{6} \mathrm{~cm}^{2} / \mathrm{Vs}$ and results in sharp peaks on the imaginary axis. The three poles with an imaginary part between 4 and $10 \mathrm{THz}$ correspond to the three peaks of the DD model
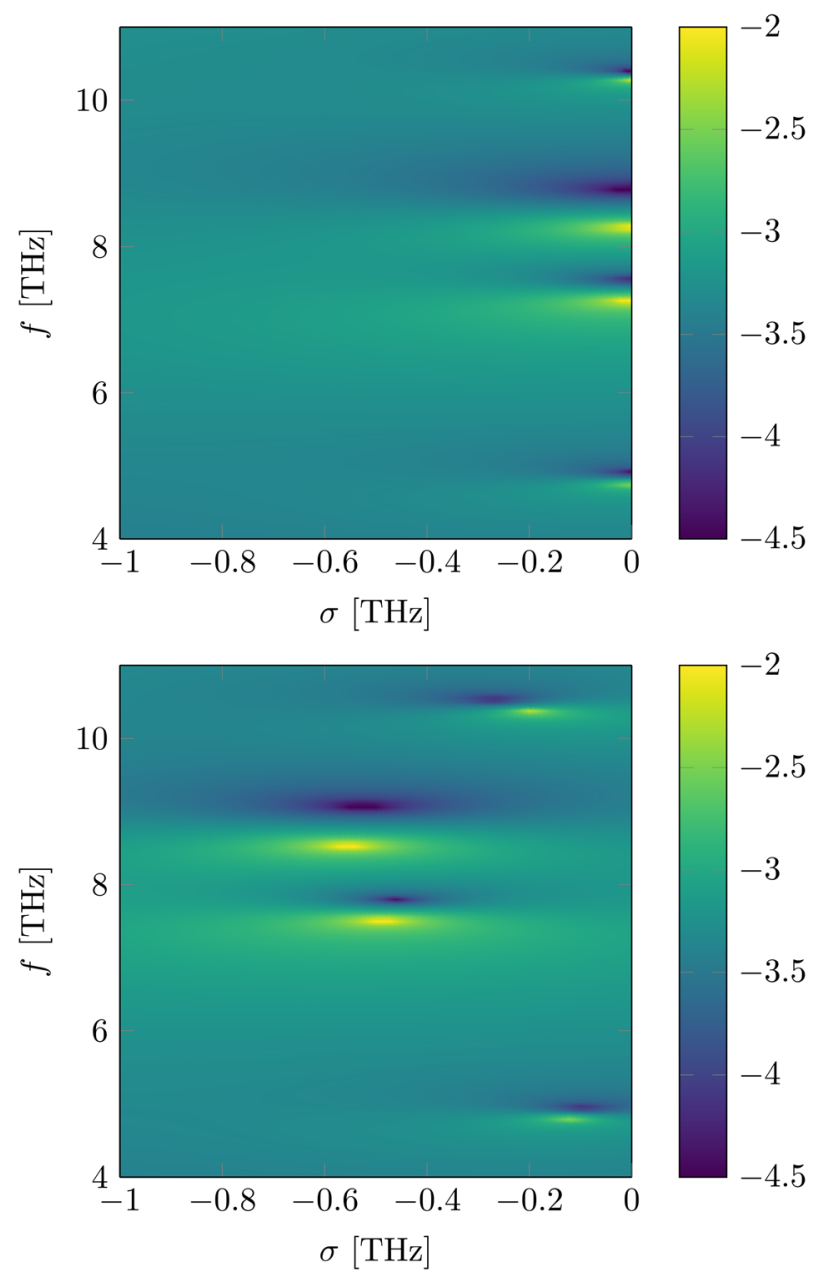

Fig. 11 Logarithm of the absolute value of the drain self-admittance for $V_{\mathrm{GS}}=0.5 \mathrm{~V}$ and $V_{\mathrm{DS}}=0 \mathrm{~V}$ (i.e., equilibrium) for $\mu=10^{6} \mathrm{~cm}^{2} /$ Vs. The results are calculated by the DD model with $\mathrm{D}-\mathrm{BCs}$ (upper figure) and TB-BCs (lower figure)

in Fig. 10 in that frequency range. On the other hand, TBBCs shift these poles (and zeros) to the left and the plasma resonances are strongly damped. This reduces their impact on the self-admittance at $\sigma=0$ which is why the results in Fig. 9 are much smoother than in Fig. 10. For a plasma instability poles or zeros with $\sigma>0$ are required. Unfortunately, the more realistic TB-BCs move the poles and zeros far to the left making plasma instabilities even more improbable. Since the DD model fails for quasi-ballistic transport anyway, the absolute values of the drain self-admittance calculated by the BE are shown in Fig. 12. The poles and zeros of the $\mathrm{BE}$ also occur at large negative real parts of $s$, and even a nonzero drain/source bias does not result in a significant shift to the right.

In Fig. 13, the nonequilibrium behavior of $\mathfrak{R}\left\{\underline{Y}_{\mathrm{DD}}(\mathrm{i} 2 \pi f)\right\}$ is shown for various drain/source voltages as a function of frequency. The BE results are compared to the tenth-order model. As it is evident at zero frequency, the 

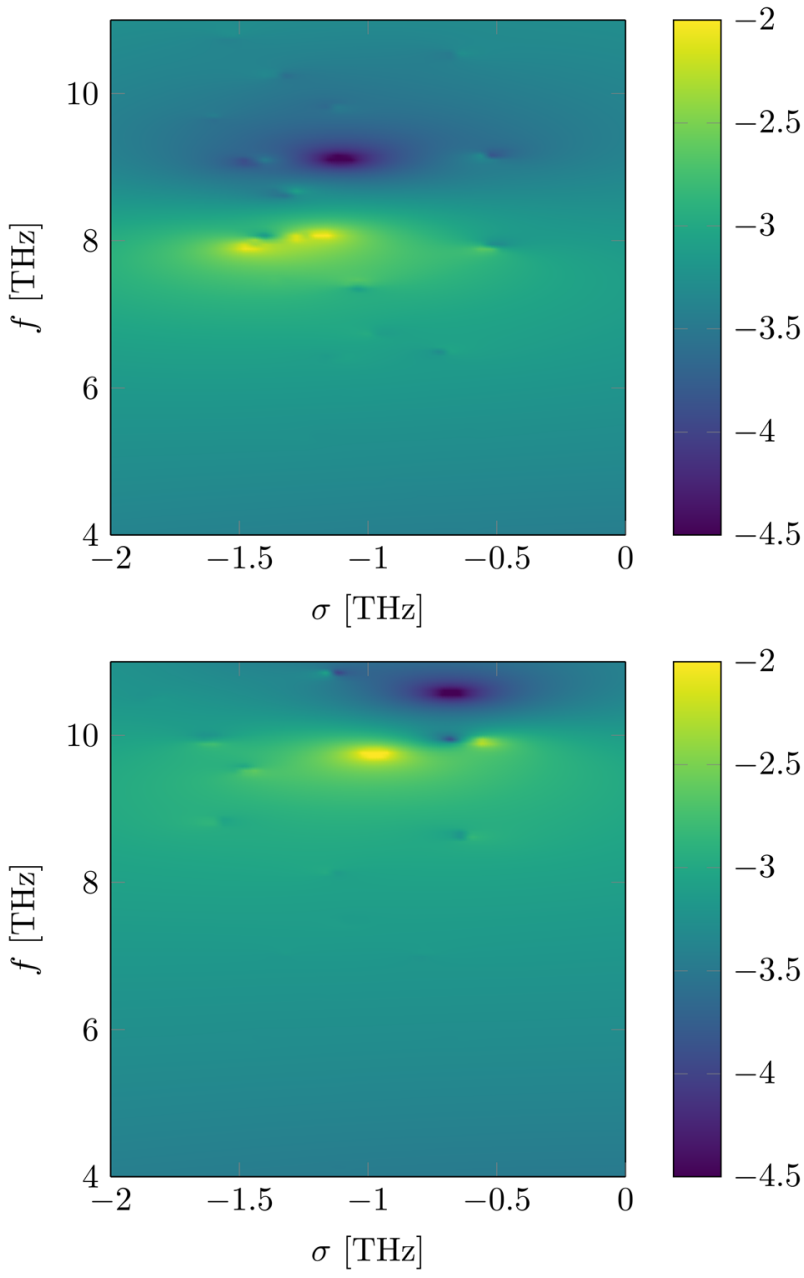

Fig. 12 Logarithm of the absolute value of the drain self-admittance for $V_{\mathrm{GS}}=0.5 \mathrm{~V}$ and the ballistic case. The results are calculated by the $\mathrm{BE}$ for $V_{\mathrm{DS}}=0 \mathrm{~V}$ (upper figure) and $V_{\mathrm{DS}}=0.1 \mathrm{~V}$ (lower figure)

transistor operates in the saturation regime for $V_{\mathrm{DS}}>0.2 \mathrm{~V}$ $\left(Y_{\mathrm{DD}}(0) \rightarrow 0\right)$. Increasing the drain bias shifts the peak at $f=8 \mathrm{TH}_{\mathrm{z}}$ to higher frequencies (see Fig. 12). The momentsbased model follows this behavior up to $V_{\mathrm{DS}}=0.1 \mathrm{~V}$, whereas for higher biases it becomes unstable and cannot produce meaningful results. The $\mathrm{BE}$, on the other hand, can be solved without problems for larger voltages and its results do not show any active behavior (i.e., $\mathfrak{R}\left\{\underline{Y}_{\mathrm{DD}}\right\}<0$ ) for drain voltages larger than zero. The above results clearly show that the internal contact BCs of the transport model play an important role and that the more realistic TB-BCs strongly damp plasma resonances.

Since the damping of the plasma resonances depends on the type of the $\mathrm{BCs}$, it would be interesting to investigate the impact of the D-BCs onto the BE results. The D-BCs are applied in the case of the moments-based models to the even moments (density, energy density (temperature),

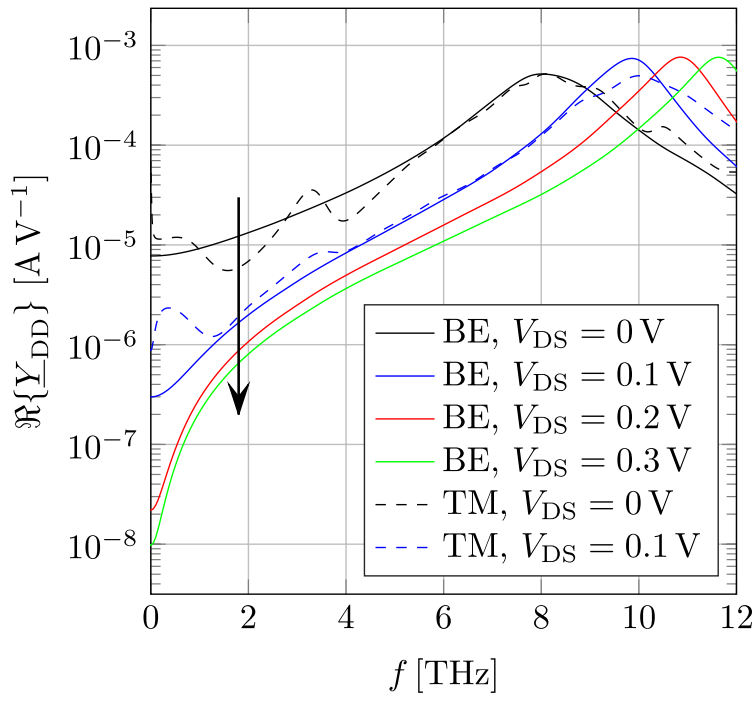

Fig. $13 \Re\left\{\underline{Y}_{\mathrm{DD}}\right\}$ versus frequency at $V_{\mathrm{GS}}=0.5 \mathrm{~V}$ using the $\mathrm{BE}$ and tenth-order moments-based model. Simulations are performed for the mobility of $\mu=10^{6} \mathrm{~cm}^{2} / \mathrm{V}_{\mathrm{s}}$ and TB-BCs. The arrow denotes the increase in $V_{\mathrm{DS}}$

etc.). In the case of the $\mathrm{BE}$, this would correspond to the application of D-BCs to the even parts of the distribution functions. Without scattering, such a boundary condition violates the Liouville theorem, because the ratio of the even part of the distribution function at the source and drain at energies above the top of the barrier is fixed by transport for an inversion symmetric bandstructure and cannot be imposed by the BCs. Thus, D-BCs for the even part of the distribution function and the ballistic BE are incompatible.

\section{Conclusion}

In this paper, a numerically stable approach for phase space discretization of the $\mathrm{BE}$ was proposed, which is applicable to the diffusive as well as ballistic regime. The presented BE solver was used to calculate the small-signal self-admittance of an $\mathrm{N}^{+} \mathrm{NN}^{+}$nanowire transistor up to $\mathrm{THz}$ frequencies, and the results were compared to the ones by moments-based models. D-BCs were shown to result in spurious plasma resonances for weak scattering, and moments-based models fail at describing the smallsignal behavior of quasi-ballistic devices. This calls into question the credibility of DD or higher-order models for the investigation of high-mobility phenomena in electronic devices. 
Acknowledgements Open Access funding provided by Projekt DEAL. Funding by the Deutsche Forschungsgemeinschaft (Ref. No.: JU406/14-1) is gratefully acknowledged.

Open Access This article is licensed under a Creative Commons Attribution 4.0 International License, which permits use, sharing, adaptation, distribution and reproduction in any medium or format, as long as you give appropriate credit to the original author(s) and the source, provide a link to the Creative Commons licence, and indicate if changes were made. The images or other third party material in this article are included in the article's Creative Commons licence, unless indicated otherwise in a credit line to the material. If material is not included in the article's Creative Commons licence and your intended use is not permitted by statutory regulation or exceeds the permitted use, you will need to obtain permission directly from the copyright holder. To view a copy of this licence, visit http://creativecommons.org/licenses/by/4.0/.

\section{References}

1. Otsuji, T., Shur, M.: Terahertz plasmonics: good results and great expectations. IEEE Microw. Mag. 15(7), 43 (2014)

2. Li, D., Zhang, L., Su, J.: Investigation of the Dyakonov-Shur instability for $\mathrm{THz}$ plasma waves in quantum gated cylindrical FET. AIP Adv. 9, 125126 (2019)

3. Li, D., Zhang, L., Du, H.: The instability of terahertz plasma waves in cylindrical FET. Plasma Sci. Technol 21(4), 045002 (2019)

4. Shur, M.: Si, SiGe, InP, III-N, and p-diamond FETs and HBTs for sub-terahertz and terahertz applications. In: Proceedings of the SPIE 11279, Terahertz, RF, Millimeter, and Submillimeter-Wave Technology and Applications XIII, p. 1127903 (2020)

5. Pan, Y. et al.: The interaction between two-dimensional electron gas and terahertz plasma wave in HEMT-like structure. In: International Conference on Infrared, Millimeter, and Terahertz Waves (IRMMW-THz), p. 1 (2019)

6. Dyakonov, M., Shur, M.: Shallow water analogy for a ballistic field effect transistor: new mechanism of plasma wave generation by DC current. Phys. Rev. Lett. 71, 2465 (1993)

7. Dyakonov, M., Shur, M.: Plasma wave electronics: novel terahertz devices using two dimensional electron fluid. IEEE Trans. Electron Devices 43(10), 1640 (1996)

8. Dyakonov, M., Shur, M.: Detection, mixing, and frequency multiplication of terahertz radiation by two-dimensional electronic fluid. IEEE Trans. Electron Devices 43(3), 380 (1996)

9. Mahi, F.Z., et al.: Terahertz small-signal response of field-effect transistor channels. IEEE Trans. Terahertz Sci. Technol. 5(4), 584 (2015)

10. Park, J.Y., et al.: Physical analysis and design of resonant plasmawave transistors for terahertz emitters. IEEE Trans. Terahertz Sci. Technol. 5(2), 244 (2015)

11. Calderón-Munoz, W.R., et al.: Hydrodynamic instability of onedimensional electron flow in semiconductors. J. Appl. Phys. 102(2), 023703 (2007)

12. Ryzhii, V., et al.: Terahertz photomixing using plasma resonances in double-graphene layer structures. J. Appl. Phys. 113(17), 174506 (2013)

13. Hong, S.-M., Jang, J.-H.: Numerical simulation of plasma oscillation in 2-D electron gas using a periodic steady-state solver. IEEE Trans. Electron Devices 62(12), 4192 (2015)

14. Jungemann, C., et al.: On the simulation of plasma waves in HEMTs and the Dyakonov-Shur instability. In: Proceedings of the SISPAD, pp. 331-334 (2019)
15. Linn, T., et al.: Investigation of moments-based transport models applied to plasma waves and the Dyakonov-Shur instability. Semicond. Sci. Technol. 34(1), 014002 (2018)

16. Stanojević, Z., et al.: Phase-space solution of the subband Boltzmann transport equation for nano-scale TCAD. In: Proceedings of the SISPAD, p. 65, (2016)

17. Lundstrom, M.: Elementary scattering theory of the Si MOSFET. IEEE Electron Device Lett. 18(7), 361 (1997)

18. Shur, M.S.: Low ballistic mobility in submicron HEMTs. IEEE Electron Device Lett. 23(9), 511 (2002)

19. Jacoboni, C., Lugli, P.: The Monte Carlo Method for Semiconductor Device Simulation. Springer, Wien (1989)

20. Jin, S., et al.: Theoretical study of carrier transport in silicon nanowire transistors based on the multisubband Boltzmann transport equation. IEEE Trans. Electron Devices 55, 2886 (2008)

21. Noei, M., Jungemann, C.: Microscopic simulation of RF noise in junctionless nanowire transistors. J. Comput. Electron. 17(3), 986 (2018)

22. Ruić, D., Jungemann, C.: Small signal and microscopic noise simulation of an nMOSFET by a self-consistent, semi-classical and deterministic approach. In: 2015 International Conference on Simulation of Semiconductor Processes and Devices (SISPAD), pp. 20-23 (2015)

23. Ringhofer, C.: Numerical methods for the semiconductor Boltzmann equation based on spherical harmonics expansions and entropy discretizations. Transp. Theory Stat. Phys. 31(4-6), 431 (2002)

24. Gnudi, A., et al.: Two-dimensional MOSFET simulation by means of a multidimensional spherical harmonics expansion of the Boltzmann transport equation. Solid-State Electron. 36(4), 575 (1993)

25. Lundstrom, M.: Fundamentals of Carrier Transport 10 of Modular Series on Solid State Devices. Addison-Wesley, New York (1990)

26. Selberherr, S.: Analysis and Simulation of Semiconductor Devices. Springer, Wien (1984)

27. Hong, S.-M., et al.: Deterministic solvers for the Boltzmann transport equation. In: Computational Microelectronics. Springer, Wien (2011)

28. Lucci, L., et al.: Multisubband Monte Carlo study of transport, quantization, and electron-gas degeneration in ultrathin SOI n-MOSFETs. IEEE Trans. Electron Devices 54, 1156 (2007)

29. Ando, T., et al.: Electronic properties of two-dimensional systems. Rev. Mod. Phys. 54, 437 (1982)

30. Ruić, D., Jungemann, C.: Numerical aspects of noise simulation in MOSFETs by a Langevin-Boltzmann solver. J. Comput. Electron. 14(1), 21 (2015)

31. Madelung, O.: Introduction to Solid State Theory. Springer, Berlin (1978)

32. Schenk, O., Gartner, K.: Solving unsymmetric sparse systems of linear equations with PARDISO. J. Future Gener. Comput. Syst. 20(3), 475 (2004)

33. Polizzi, E.: Density-matrix-based algorithm for solving eigenvalue problems. Phys. Rev. B 79, 115112 (2009)

34. Jin, S., et al.: Simulation of silicon nanowire transistors using Boltzmann transport equation under relaxation time approximation. IEEE Trans. Electron Devices 55(3), 727 (2008)

35. Moler, C., Van Loan, C.: Nineteen dubious ways to compute the exponential of a matrix, twenty-five years later. SIAM Rev. 45(1), 3 (2003)

36. Sidje, R.B.: Expokit: a software package for computing matrix exponentials. ACM Trans. Math. Softw. (TOMS) 24(1), 130 (1998)

37. Kim, H., et al.: An extended proof of the Ramo-Shockley theorem. Solid-State Electron. 34, 1251 (1991) 
38. Esseni, D., et al.: Nanoscale MOS Transistors, Semi-Classical Transport and Applications. Cambridge University Press, Cambridge (2011)

39. Gonzalez, G.: Microwave Transistor Amplifiers: Analysis and Design, 2nd edn. Prentice-Hall, Upper Saddle River (1997)

40. Nekovee, M., et al.: Failure of extended-moment-equation approaches to describe ballistic transport in submicrometer structures. Phys. Rev. B 45(12), 6643 (1992)

41. Jungemann, C., et al.: Failure of moments-based transport models in nanoscale devices near equilibrium. IEEE Trans. Electron Devices 52(11), 2404 (2005)
Publisher's Note Springer Nature remains neutral with regard to jurisdictional claims in published maps and institutional affiliations. 